\title{
A Review on Machine Learning-Based Radio Direction Finding
}

\author{
Ming-Yi You $\mathbb{D}^{1,2}$ An-Nan Lu, ${ }^{1,2}$ Yun-Xia Ye $\mathbb{D}^{1,2}$ Kai Huang $\mathbb{D}^{1,2}$ and Bin Jiang ${ }^{1,2}$ \\ ${ }^{1}$ Science and Technology on Communication Information Security Control Laboratory, Jiaxing, Zhejiang 314033, China \\ ${ }^{2}$ No. 36 Research Institute of CETC, Jiaxing, Zhejiang 314033, China
}

Correspondence should be addressed to Ming-Yi You; youmingyi@126.com

Received 1 July 2020; Accepted 17 August 2020; Published 24 August 2020

Guest Editor: Jianfeng Li

Copyright (C) 2020 Ming-Yi You et al. This is an open access article distributed under the Creative Commons Attribution License, which permits unrestricted use, distribution, and reproduction in any medium, provided the original work is properly cited.

The research and applications of radio direction-finding technology based on machine learning are reviewed. Detailed application scenarios are summarized with focus on the advantages of machine learning-based direction-finding models. Important elements such as problem formulation and model inputs and outputs are introduced in detail. Finally, some valuable future research topics are discussed.

\section{Introduction}

Radio direction finding is the measurement of the direction from which a received signal is transmitted. Recently, direction-finding technology has been widely applied in emitter localization, jamming guide, determination of communication relationship, communication network division, cooperative communication, etc. Radio direction finding is essentially an inverse problem of signal reception from a given direction, and the core is the mapping relationship of several signal parameters with its incidence direction.

With the advances in machine learning and artificial intelligence, some researchers gradually began to consider the problem of direction finding using machine learning techniques. The idea of direction finding using machine learning techniques can be dated back to the 1990s. At that time, some researchers considered the application of artificial neural networks (ANNs) to direction finding with fast development in different types of shallow neural networks. At about 2000s, support vector machines (SVMs) achieved satisfactory results in many fields including direction finding. Recently, deep learning techniques greatly improve the modeling capability of neural networks and are attracting more and more researchers and engineers in the field of direction finding.

With wide applications of machine learning techniques to direction finding problems, however, a systematic overview that collects, organizes, clarifies, and compares the related works has not yet been reported. By this motivation, facing the applications of machine learning techniques in direction finding, the main objective of the paper is to collect and extract the specific problems solved by machine learning models, to summarize the main types of model inputs and outputs, and to raise several problems that deserve further research.

\section{Research and Applications of Machine Learning Techniques in Direction Finding}

In general, machine learning techniques have special advantages for direction finding in complex scenarios compared with classical approaches such as analytical methods and the least square (LS) method. Such complex scenarios include complex receiving systems, complex channels, and complex signals. In addition, machine learning techniques are usually applied to improve direction finding capabilities such as computing speed and angle resolution. Figure 1 summarizes the direction finding scenarios where machine learning techniques have been utilized. In the following, we will review the research works that could be categorized into the corresponding application scenarios, with focus on the problems to be solved and the model inputs and outputs. 


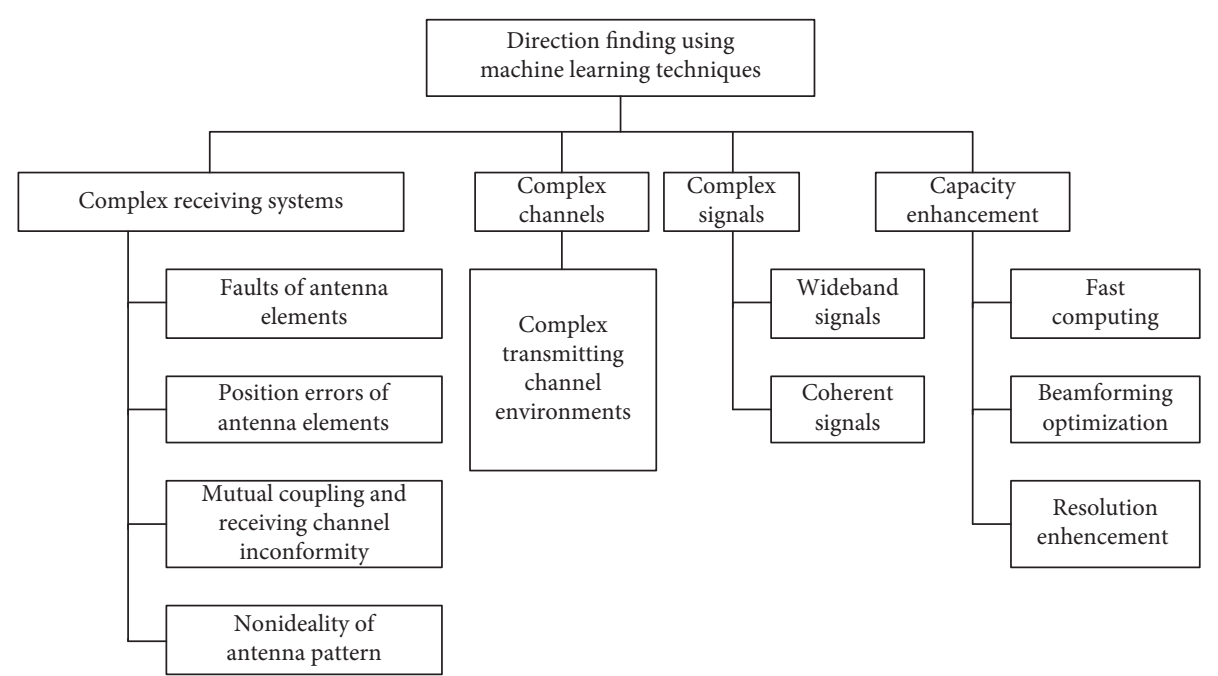

FIGURE 1: Overview of applications of machine learning techniques in direction finding.

\subsection{Concrete Scenarios}

2.1.1. Complex Receiving Systems. Scenarios of complex receiving systems include the scenarios where there are position errors of antenna elements, the scenarios where there are mutual coupling of antenna elements and receiving channel inconformity, the scenarios of nonideal antenna pattern, and the scenarios where some antenna elements malfunction.

Shieh and Lin [1] pointed out that high resolution methods such as multiple signal classification (MUSIC) were highly sensitive to the structure of the covariance matrix and required excessively large computation effort. The estimation of signal parameters by rotational invariance techniques (ESPRIT) was faster at the expense of increased number of sensors. Both MUSIC and ESPRIT algorithms were very sensitive to imperfections of models of signals and noise. To handle the abovementioned problem, a neural fuzzy scheme was proposed in [1] based on the phase differences from an interferometer. A self-constructing neural fuzzy inference network (SONFIN) was used in [1] which was a general connectionist model of a fuzzy logic system and could find its optimal structure and parameters automatically. In addition, to avoid the discontinuities caused by the input phase transition, a quadrature representation of the phase differences was used as the input of SONFIN. The output of SONFIN is the direction of arrival (DOA). Simulation results showed that the SONFIN always produced DOA estimates close to the desired DOA values, and the required number of parameters of SONFIN was much less than that in classical radial basis function network (RBFN) under the same root mean square error in DOA.

Motivated by the fact that once an antenna is built and placed on a certain platform, changes that affect both the shape of the structure and effectiveness of the materials used to fabricate the antenna may occur. Christodoulou et al. [2] proposed a least square support vector machine (LSSVM) approach to direction finding to make arrays smart so that the beamforming and beamsteering performance of the array degraded gracefully when one of the antenna elements failed. The LSSVM algorithm was trained with projection vectors generated from the signal subspace eigenvectors and the respective covariance matrices. And the output labels from the multiclass LSSVM system were the DOA estimates. Rohwer et al. [3] adopted a similar approach for DOA estimation. Following the works by Rohwer et al. [3], an extensive sensitivity analysis was carried out in [4] to understand how parameters of the LSSVM formulation affect the performance of the resulting multiclass classifier system, with a clear dependence on the width of the radial basis kernel function.

Motivated by the requirement of not monitoring the failed antenna element and time of failure, S. Vigneshwaran et al. [5] proposed a direction-finding approach based on the minimal resource allocation network (MRAN). The training for MRAN was conducted under no failure and no noise cases and the trained network was then used when there was a failure. To overcome the problem of high direction finding error near $\pm 90^{\circ}$, Vigneshwaran et al. [5] used the magnitude and phase angles of the elements of the correlation matrix instead of their real and imaginary parts as the network inputs. During the training process, MRAN has larger number of tunable parameters as it tunes the centers, weights, and spread of the neurons, whereas the classical RBFN tunes just the weights. And this leads to higher generalization capability of MARN.

Dehghanpour et al. [6] dealt with direction finding in presence of mutual coupling of antenna elements using a multiple kernel SVM. The normalized elements of the upper triangle part of the correlation matrix were used as the inputs of the multiple kernel SVM, whereas the DOAs are used as the model outputs. Simulation results demonstrated the superiority of the proposed method over classical methods such as MUSIC, single kernel support vector regression (SVR), and smooth SVR.

Wang et al. [7] pointed out that the performance of MUSIC algorithm relied on the precision of the antenna pattern which could be contaminated by nearby 
electromagnetic interference and was not easy to measure, and therefore it was meaningful to seek a direction finding approach without the need of precise measurement of the antenna pattern. They developed a SVR-based direction finding method for an M-element radar receiving system. The relative magnitude and phase of signals to those of the reference receiving channel were used as the model input. The field experiments conducted in Pingtan and Liuao validated that the SVR-based method outperformed the classical MUSIC algorithm.

Liu et al. [8] pointed out that the existing learning-based direction finding methods suffered the following drawbacks: (1) the generalization capability was questionable where the volume of the training set was limited; (2) the existing methods were mainly applicable to the case of single object and could not be applied to a general direction-finding problem when the number of objects was unknown. They proposed a hierarchical framework of deep NNs (DNNs) to deal with the general DOA estimation problem. The framework consists of an autoencoder for spatial filtering and a multiclass classifier for DOA estimation. The normalized elements in the upper triangle part of the correlation matrix were used as the model input. The simulation results showed that the proposed method was applicable to the scenarios when the number of incident directions differed from those of training samples.

2.1.2. Complex Channels. Research on machine learningbased direction finding in complex transmitting channels mainly focuses on indoor direction finding and localization in presence of multipath effect and heavy reverberation.

Xiao et al. [9] pointed out existing direction finding approaches faced either one or a combination of the following problems: high-computational cost, nonrealistic assumptions on signal/noise models, and unreliable performance in real environments. To estimate DOAs in noisy and reverberate environments, they formulated the task of direction finding as a classification problem, extracted features from the generalized cross correlation (GCC) vectors, and used a multilayer perception (MLP) NN to learn the nonlinear mapping from such features to the DOA. Experimental results on simulated data showed that the method performed much better than the LS method.

To address the problem of localization in adverse environments such as high reverberation and low signal-tonoise ratio (SNR), Sun et al. [10] proposed an indoor sound source localization approach with a probabilistic NN (PNN). The GCC features were again used as the network inputs, whereas the space of interest was divided into a number of equal-dimensional rectangular clusters.

Cheng et al. [11] compared the capability of several SVMs and the k-means approach for indoor localization using the magnitudes of ambient FM and DVB-T signals. The comparison results demonstrated the superiority of the SVMs. In addition, Cheng et al. [11] discussed the problem of feature extraction and ensemble learning.

Yue et al. [12] pointed out that traditional signal processing methods could not handle noise and reverberation effectively and proposed a learning-based approach of DOA estimation in a three-dimensional room space. GCC with phase transform (GCC-PHAT) was used as the input to a convolutional $\mathrm{NN}(\mathrm{CNN})$, and it was formulated as a classification problem. Accuracy of single sound source direction detection and compatibility of multiple sound sources were verified by extensive simulations.

$\mathrm{Li}$ et al. [13] proposed a method combining CNN and long short-term memory (LSTM) network to address the online DOA estimation in noisy and reverberant environments. The proposed approach used a two-dimensional input matrix spreading over the time and frequency domain for GCC-PHAT. In addition, the feature matrixes for all sensor pairs were summed up for robustness improvement. Experimental results showed that the method was robust to the topologies of microphone array and the trained model could get better performance on a new microphone array structure using only very few new data for adaptation.

Mane et al. [14] proposed a CNN-based classification method for broadband DOA estimation of a single steady sound source in noisy and reverberation conditions. The input to the CNN was given as the short-time Fourier transform (STFT) coefficients of the phase components obtained from the uniform linear array (ULA) of microphones. Similar approach was suggested in [15] with extension to multiple sources with the assumption of disjoint speaker activity in STFT domain.

Khan et al. [16] pointed out that the MUSIC spectrum would not produce a clean outstanding peak in the case of multipath and proposed a NN-based DOA estimation model with the MUSIC spectrum as the input. The performance of the proposed model showed consistent improvement over a range of channel parameters including elevation angles, single-to-noise ratios (SNRs), and channel configuration.

Fahim et al. [17] proposed a multisource DOA estimation technique using a $\mathrm{CNN}$ algorithm which learnt the modal coherence patterns of an incident sound field through measured spherical harmonic coefficients. The model was trained with individual time-frequency bins in the STFT spectrum. The proposed model was capable of estimating multiple sound sources on a three-dimensional space using a single-source training set.

2.1.3. Complex Signals. Wideband signals and coherent signals impose special difficulties for accurate direction finding. Recently, some researchers tried to solve the problem of DOA estimation of wideband or coherent signals using machine learning techniques.

A novel class of focusing matrices for coherent signal subspace method was proposed in [18]. These matrices are formed based on a backpropagation (BP) $\mathrm{NN}$ and the concept of uniform focusing transformation of the DOA of wideband sources. The proposed DOA estimating procedure is efficient without prior knowledge of the DOAs.

Lizzi et al. [19] presented an approach for DOA estimation of correlated signals based on a SVM classifier and an iterative multiscaling approach (IMSA). The use of SVM guarantees real-time operations, while the IMSA enhances 
the angular resolution within the regions of incidence of the incoming signals.

Terabayashi et al. [20] proposed a DOA estimation method using a complex-valued spatiotemporal NN (CVSTNN) for ultrawideband (UWB) systems. The CVSTNN was combined with the power-inversion adaptive array (PIAA) for null steering without the knowledge of incident directions. Simulation and experimental results demonstrated that the CVSTNN showed a higher accuracy than the conventional MUSIC method.

A fast DOA estimation method based on CNN for broadband radio direction finding system was proposed in [21]. The phase component of the covariance matrix was fed into the network, whereas each DOA label was consisted of the sine and cosine values of the angel of arrival (AOA).

Mack et al. [22] extended broadband DOA estimation to broadband signal-aware DOA estimation by focusing on narrow frequency bands dominated by the desired source and removing the spatial information in other bands. The experiments with estimated masks showed the possibility to combine signal-independent DOA with independently trained mask estimators to perform signal-aware DOA estimation.

2.1.4. Capability Enhancement. Capability enhancement is continuously a core topic for research on direction finding systems. With the help of machine learning techniques, researchers have made progress on weight parameter optimization for beamforming, computation acceleration, and direction finding resolution improvement.

The 1st part of capability enhancement is beamforming optimization. $\mathrm{Xu}$ et al. [23] proposed a beamforming algorithm using SVM. The sampled signals were used as the model input, whereas the expected signal sequences served as the model output.

Savitha et al. [24] compared the performance of beamforming using the fully complex-valuved RBF (FC$\mathrm{RBF}$ ) network with the fully complex-valued activation function with the performance of the existing complexvalued RBF network. The received signals were used as the model input and the expected transmitted signals were used as the model output.

One of smart antennas' main functions is adaptive beamforming, which forms a high-gain beam at the desirable signal directions and weakens interference signals in the other directions. However, the real-time calculation of adaptive weighting coefficients is computationally intensive, especially in the case of a large number of antenna array elements. Hence, Pei et al. [25] proposed an efficient method for real-time calculation of beamforming weighting coefficients based on the generalized regression NN. The normalized elements of the upper triangle part of the correlation matrix were used as the model input and the normalized weighting matrix was used as the model output. The simulation results verified the superiority of the proposed method over the minimum variance distortionless response (MVDR) method in terms of computing speed.
Salvati et al. [26] presented a weighted MVDR algorithm for far-field broadband sound source localization in a noisy environment. A machine learning method based on a SVM was used for selecting the narrowband components that positively contributed to the broadband fusion. The skewness measure of response power function was used as the input feature for the supervised SVM learning. Simulations demonstrated the effectiveness of the weighted MVDR in an outdoor noisy environment.

Zaharis et al. [27] introduced the implementation of antenna array beamforming based on a simplified CNN. The inputs to the CNN were the AOAs of signals of interest (SOI) and signals of avoidance (SOA), whereas the output was the expected magnitude and phase of exciting weights. In the proposed NN structure, the first hidden layer was divided into sublayers, and a direct connection between every input and a respective sublayer was performed in order to alleviate the performance degradation due to uncertainties in interference correlation.

The 2nd part of capability enhancement is fast computing. Jha and Durrani [28] pointed out that the maximum likelihood estimator was the optimal estimator of the direction of sources, but it required the minimization of a complex, multimode, and multidimensional cost function. A neural optimization procedure was presented that did not require an initial estimate of the direction of sources, which offered the potential of real-time solutions to the DOA estimation problem by utilizing the fast relaxation properties of the Hopfield network.

In 1998, Zooghby et al. [29-31] pointed out that superresolution algorithms for DOA estimation were computationally intensive and hard to implement in real time and proposed a NN-based direction finding approach. The approach was claimed to be able to track any numbers of incident directions with any angle separation without prior knowledge on the emitter number. The approach firstly divided the angle region of interest into several subregions and trained the same number of NNs to judge if the emitter belonged to a given subregion with the normalized upper triangle part of the covariance matrix as the model input. Afterwards, several NNs were trained to accurately estimate the DOAs in each subregion, and each subregion was further divided into $N$ girds. Simulation results demonstrated that the proposed NN could estimate different numbers of DOAs from those of the training samples. They also considered using the DOAs as the NN output directly [32, 33]. Similar inputs are considered in $[34,35]$, except that Pour et al. [34] used a MLP network along with ant colony optimization for $\mathrm{NN}$ training and a BP NN was used in [35]. A similar treatment for a uniform linear array was adopted in [36].

Jeong et al. [37] addressed the problem for the DOA estimation of narrowband emitter signals impinging on an array of sensors by the modified Hopfield NN. They showed that the operation of the $\mathrm{NN}$ had an analog with the covariance fit estimator in estimating the DOA.

To reduce the computation complexity, Tong et al. [38] studied satellite interference location through a RBF network. The time averaged and normalized signals were used 
as the model input, whereas the DOAs were designed as the model output.

Based on a four-element linear array, Lee [39] compared the performance of three types of NNs for direction finding, namely, a Hopfield NN, a BP NN, and a RBF NN. He pointed out that appropriately choosing the center would accelerate the training process of the RBF NN.

Chen et al. [40] proposed a method for reducing the volume of training samples for two-dimensional direction finding using a uniform circular array. The elevation and azimuth were estimated in two separate NNs, respectively. A similar strategy for two-dimensional DOA estimation was adopted in [41] based on a linear vector quantization (LVQ) NN.

Matsumoto and Kuwahara [42] conducted a direction finding experiment using a RBF NN. To avoid the discontinuity near $0^{\circ}$, the outputs of the $\mathrm{NN}$ were designed as $[\sin \alpha$, $\cos \alpha$ ], where $\alpha$ was the AOA. The input of the $\mathrm{NN}$ is a vector composed of the normalized elements of the upper triangle part of the covariance matrix.

Raj et al. [43] pointed out that there were two main disadvantages of the direction-finding algorithms such as MUSIC, root-MUSIC, and ESPRIT. Firstly, they are computational intensive. And secondly, they assume that the antenna elements are exactly equal. Raj et al. [43] used a nonlinear SVR approach for direction finding where the fixed number of angles and their corresponding normalized array input vectors were used as the output/input pair.

Gotsis et al. [44, 45] proposed a DOA estimation methodology based on NNs and designed for a switchedbeam system. The method incorporated the benefits of NNs and switch-beam systems to achieve DOA estimation in a less complex and expensive way compared to the corresponding widely known super-resolution algorithms. The $\mathrm{NN}$ firstly despreaded direct sequence code division multiple access (DS-CDMA) signals and the signal amplitudes of multiple beams were used as the model inputs. It was shown that a properly trained $\mathrm{NN}$ could accurately find the SOI $\mathrm{AOA}$ at the presence of a varying number of mobile users and a varying SOI to interference ratio. Similar inputs were used in [46] to design a compact DOA estimation system which used only four circularly patch elements. NNs were used to firstly classify the antenna signals and then estimate the DOA.

George and Sajjanshetty [47] proposed a technique for the estimation of DOA of moving signals using two architectures of NNs, i.e., feedforward and RBF NNs. A fundamental assumption made was that the number of signals was either known, or could be estimated in priori. The normalized sensor array output was given as the input to the network.

Agatonovic et al. [48-51] proposed a two-level NN for two-dimensional direction finding. The first-level $\mathrm{NN}$ classified the AOA into a small subregion, whereas the second-level $\mathrm{NN}$ estimated the AOA accurately. The inputs to the two NNs were the normalized elements of the first array of the covariance matrix. To deal with the mutual coupling among antenna elements, similar methods are adopted in [52-54]. Similar features are used in [55] for DOA estimation of a multiple input multiple output
(MIMO) orthogonal frequency division multiplexing (OFDM) radar.

To reduce the real-time computation burden, Stankovic et al. [56-59] proposed a direction-finding approach using a MLP NN. The inputs of the NN are all elements of the covariance matrix and the output are the DOAs. NN training was conducted for the scenarios of one emitter and two emitters with fixed DOA separation.

Chakrabarty and Habets [60] pointed out that the existing NNs used generalized correlation or covariance matrix as model inputs, and even the process of feature extraction was time consuming. They proposed a direction finding approach for wideband signals using only phase of each spectral lines. A classification NN was established for direction finding. The experimental results demonstrated that the $\mathrm{NN}$ trained by simulated data could be effectively applied in real signal environments.

Faye et al. [61] demonstrated the ability of a single uniform linear array (ULA) of isotropic elements along with an NN approach to achieve two-dimensional DOA estimation. LVQ NNs were sequentially trained on elevation and azimuth-dependent datasets built from received signal in predefined spatial sectors chosen in accordance with pattern symmetry and radiation intensity.

Efimov et al. [62] presented the approach to the design of AOA estimator for narrowband noise-like signal based on $\mathrm{NN}$ to improve the signal processing speed. The signal time delay of each sensor pair was used as the model input and the associated AOA was designed as the model output.

Huang et al. [63] proposed a deep learning based superresolution DOA estimation model in the uplink MIMO system with the model input and output to be the array received signals and the DOA, respectively. Similar treatment was adopted in [64] for sound source localization and direction finding with a CNN.

The 3rd part of capability enhancement is resolution improvement. Wang and Ma [65] compared the estimation bias and angle resolution of a NN-based direction finding approach with MUSIC when signals were uncorrelated, partially correlated, and correlated.

Chen and Hou [66] proposed a principle component decomposition approach based on a complex-valued NN. The estimated feature vectors from the NN were used for direction finding based on MUSIC to obtain high resolution estimations. Similarly, Chang et al. [67] proposed a high resolution bearing estimation method via unitary decomposition artificial NN.

A DOA estimation method based on a cascaded $\mathrm{NN}$ was proposed in [68] for two closely spaced sources. The NN contained two parts: SNR classification network and DOA estimation network. Due to the cascade structure, the method could be applied to a wider range of SNR than other existing algorithms.

\section{Summary}

Based on the reported studies, Figure 2 summarizes the commonly used inputs, models, and outputs for machine learning-based direction finding. 


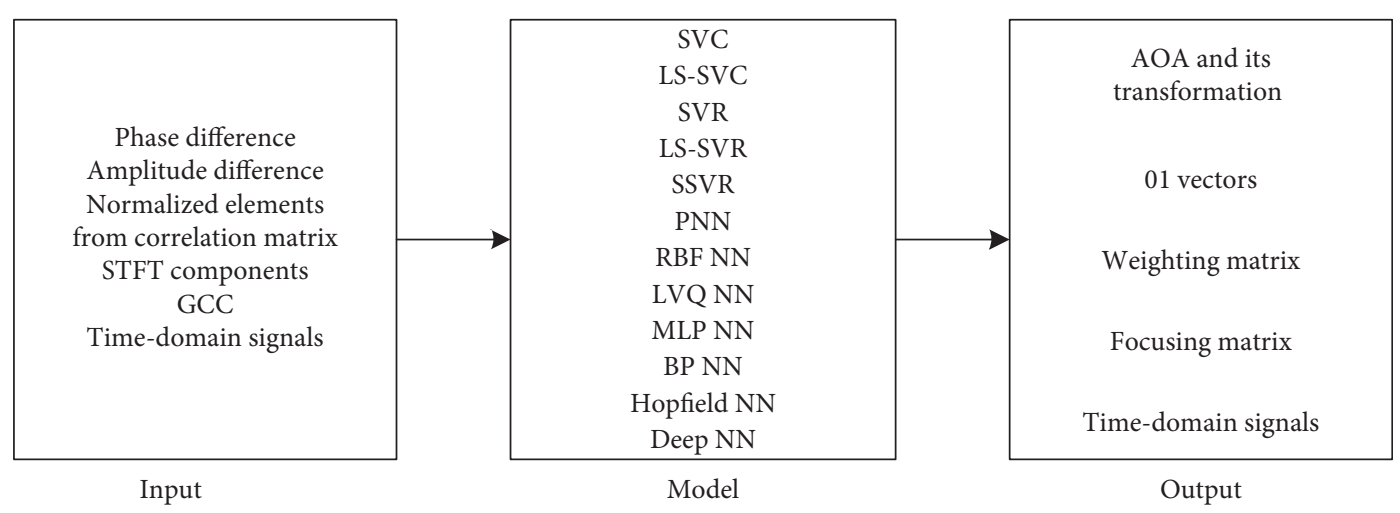

Figure 2: Commonly used inputs, models, and outputs for machine learning-based direction finding.

\section{Future Challenges}

Based on the above review, it is evident that machine learning techniques have been widely used in direction finding. However, there are still many challenges when these intelligent models are applied to practical engineering problems.

The first problem in performing two-dimensional direction finding is the huge volume of training samples which prohibits practical use of NN models of direction finding. Chen et al. [40] has suggested a method for reducing the necessity for training samples for a uniform circular array. However, an effective training method is still an open issue for general array formulations.

Section 2 has summarized the common features for machine learning models. However, it is still unclear which type is most effective. Deep learning techniques may shed light on this problem by feeding original signal samples into a DNN and letting the network learn the feature vectors by itself.

In the application process of machine learning-based direction-finding approaches, more training samples can be collected. It might be interesting to study the incremental learning method for direction finding which could quickly update the model capability without batch processing.

For the scenario where elements of an antenna array fails and the failure time is unknown, Vigneshwaran et al. [5] suggests an MRAN-based method, but the capability loss is still remarkable. In engineering applications, it is common to see failures or degradation of antenna elements, and it would be beneficial to establish a more robust direction-finding system by considering the possibility of element failure even in the training phase of the NN.

In some cases such as field calibration of satellite antennas, the AOAs may even be uncertain to some extent for the training samples, and how to establish the mapping between the extracted features and the AOAs remains an unsolved problem.

To solve the problem of direction finding when the AOAs of a testing sample are different from those in the training samples [8], it is common to use 01 vector as the model output. It is recognized that a $\mathrm{NN}$ for general direction finding is necessary to deal with the problem effectively, but it is still unclear whether there are other effective modeling forms of the NN output.

Most current works consider the case of direction finding for a single central frequency explicitly or inexplicitly. For practical cases when multiple central frequencies are of interest, the frequency information must be considered which may significantly influence array manifold. Establishing a NN separately for each central frequency may be very time consuming and hard to implement in real time; hence, it is necessary to establish a unified NN which could handle direction finding for multiple central frequencies.

\section{Concluding Remarks}

The research and applications of machine learning-based direction finding are reviewed and discussed, with focus on application scenarios, concrete problems to be solved, and model inputs and outputs. Some future challenges and interesting research topics are also discussed. It has been twenty years since the initial use of machine learning techniques for direction finding, and it is expected that direction finding systems have better performance in more and more complex application scenarios with rapid development of dig data and deep learning techniques. And this all depends on continuous research on related theories and applications.

\section{Conflicts of Interest}

The authors declare that they have no conflicts of interest.

\section{Acknowledgments}

This work was support by No. 36 Research Institute of CETC under the project no. CX05.

\section{References}

[1] C.-S. Shieh and C.-T. Lin, "Direction of arrival estimation based on phase differences using neural fuzzy network," IEEE Transactions on Antennas and Propagation, vol. 48, no. 7, pp. 1115-1124, 2000.

[2] C. G. Christodoulou, J. A. Rohwer, and C. T. Abdallah, “The use of machine learning in smart antennas,"vol. 1, pp. 321324, in Proceedings of the IEEE Antennas and Propagation 
Society Symposium, vol. 1, pp. 321-324, IEEE, Monterey, CA, USA, June 2004.

[3] J. A. Rohwer, C. T. Abdallah, and C. G. Christodoulou, "Least squares support vector machines for direction of arrival estimation with error control and validation," in Proceedings of the GLOBECOM 2003, pp. 2172-2176, San Francisco, CA, USA, December 2003.

[4] C. A. M. Lima, C. Junqueira, R. Suyama, F. J. V. Zuben, and J. M. T. Romano, "Least-square support vector machines for DoA estimation: a step-by-step description and sensitivity analysis," in Proceedings of International Joint Conference on Neural Networks, pp. 3226-3231, IEEE, Montreal, Canada, July-August 2015.

[5] S. Vigneshwaran, N. Sundararajan, and P. Saratchandran, "Direction of arrival (DoA) estimation under array sensor failures using a minimal resource allocation neural network," IEEE Transactions on Antennas and Propagation, vol. 55, no. 2, pp. 334-343, 2007.

[6] M. Dehghanpour, V. T. T. Vakili, and A. Farrokhi, "DoA estimation using multiple kernel learning SVM considering mutual coupling," in Proceedings of the 2012 Fourth International Conference on Intelligent Networking and Collaborative Systems, IEEE, Bucharest, Romania, pp. 55-61, September 2012.

[7] R. Wang, B. Wen, and W. Huang, "A support vector regression-based method for target direction of arrival estimation from HF radar data," IEEE Geoscience and Remote Sensing Letters, vol. 15, no. 5, pp. 674-678, 2018.

[8] Z.-M. Liu, C. Zhang, and P. S. Yu, "Direction-of-arrival estimation based on deep neural networks with robustness to array imperfections," IEEE Transactions on Antennas and Propagation, vol. 66, no. 12, pp. 7315-7327, 2018.

[9] X. Xiao, S. Zhao, X. Zhong, D. L. Jones, E. S. Chng, and H. Li, "A learning-based approach to direction of arrival estimation in noisy and reverberant environments," in Proceedings of the ICASSP, IEEE, Brisbane, Australia, pp. 2814-2818, April 2015.

[10] Y. Sun, J. Chen, C. Yuen, and S. Rahardja, "Indoor sound source localization with probabilistic neural network," IEEE Transactions on Industrial Electronics, vol. 65, no. 8, pp. 6403-6413, 2018.

[11] Y.-K. Cheng, R. Y. Chang, and L.-J. Chen, "A comparative study of machine-learning indoor localization using FM and DVB-T signals in real testbed environments," in Proceedings of the 2017 IEEE 85th Vehicular Technology Conference, IEEE, Sydney, Australia, pp. 1-7, June 2017.

[12] X. Yue, G. Qu, B. Liu, and A. Liu, "Detection sound source direction in 3D space using convolutional neural networks," in Proceedings of the First International Conference on Artificial Intelligence for Industries, IEEE, Laguna Hills, CA, USA, pp. 81-84, September 2018.

[13] Q. Li, X. Zhang, and H. Li, "Online direction of arrival estimation based on deep learning," in Proceedings of the ICASSP 2018, IEEE, Calgary, Canada, pp. 2616-2620, April 2018.

[14] S. S. Mane, S. G. Mali, and S. P. Mahajan, "Localization of steady sound source and direction detection of moving sound source using CNN," in Proceedings of the 10th ICCCNT 2019, IEEE-45670, Kanpur, India, July 2019.

[15] S. Chakrabarty and E. A. P. Habets, "Multi-speaker DOA estimation using deep convolutional networks trained with noise signals," IEEE Journal of Selected Topics in Signal Processing, vol. 13, no. 1, pp. 8-21, 2019.

[16] A. Khan, S. Wang, and Z. Zhu, "Angle-of-arrival estimation using an adaptive machine learning framework," IEEE Communications Letters, vol. 23, no. 2, pp. 294-297, 2019.
[17] A. Fahim, P. N. Samarasinghe, and T. D. Abhayapala, "Multisource DOA estimation through pattern recognition of the modal coherence of a reverberant soundfield," IEEE/ACM Transactions on Audio, Speech, and Language Processing, vol. 28, pp. 605-618, 2020.

[18] D. L. Zhong, X. K. Huang, and S. J. Zhang, "A novel algorithm for wideband DoA estimates based on neural network,"vol. 4, pp. 2167-2170, in Proceedings of International Conference on Neural Networks, vol. 4, pp. 2167-2170, IEEE, Houston, TX, USA, June 1997.

[19] L. Lizzi, G. Oliveri, P. Rocca, and A. Massa, "Estimation of the directions-of-arrival of correlated signals by means of a SVMbased multi-resolution approach," in Proceedings of the 2010 IEEE Antennas and Propagation Society International Symposium, July 2010.

[20] K. Terabayashi, R. Natsuaki, and A. Hirose, "Ultrawideband direction-of-arrival estimation using complex-valued spatiotemporal neural networks," IEEE Transactions on Neural Networks and Learning Systems, vol. 25, no. 9, pp. 1727-1732, 2014.

[21] W. Zhu and M. Zhang, "A deep learning architecture for broadband DOA estimation," in Proceedings of the 19th IEEE International Conference on Communication Technology, pp. 244-247, IEEE, Xi'an, China, October 2019.

[22] W. Mack, U. Bharadwaj, S. Chakrabarty, and E. A. P. Habets, "Signal-aware broadband DOA estimation using attention mechanisms," in Proceedings of the ICASSP 2020, pp. 49304934, Barcelona, Spain, May 2020.

[23] N. Xu, C. G. Christodoulou, and M. Martinez-Ramon, "Antenna array processing for radar applications using support vector machines," in Proceedings of the Antennas and Propagation Society International Symposium, pp. 1295-1298, IEEE, Albuquerque, NM, USA, July 2006.

[24] R. Savitha, S. Vigneswaran, S. Suresh, and N. Sundararajan, "Adaptive beamforming using complex-valued radial basis function neural networks," in Proceedings of the TENCON, pp. 1-6, IEEE, Singapore, Singapore, January 2009.

[25] B. Pei, H. Han, Y. Sheng, and B. Qiu, "Research on smart antenna beamforming by generalized regression neural network," in Proceedings of the 2013 IEEE International Conference on Signal Processing, Communication and Computing, pp. 1-4, IEEE, KunMing, China, August 2013.

[26] D. Salvati, C. Drioli, and G. L. Foresti, "On the use of machine learning in microphone array beamforming for far-field sound source localization," in Proceedings of the 2016 IEEE International Workshop on Machine Learning for Signal Processing, pp. 1-6, Vietri sul Mare, Italy, September 2016.

[27] Z. D. Zaharis, T. V. Yioultsis, C. Skeberies et al., "Implementation of antenna array beamforming by using a novel neural network structure," in Proceedings of the 2016 International Conference on Telecommunications and Multimedia, pp. 1-5, IEEE, Heraklion, Greece, July 2016.

[28] S. Jha and T. Durrani, "Direction of arrival estimation using artificial neural networks," IEEE Transactions on Systems, Man, and Cybernetics, vol. 21, no. 5, pp. 1192-1201, 1991.

[29] A. H. E. Zooghby, C. G. Christodoulou, and M. Georgiopoulos, "A neural network-based smart antenna for multiple source tracking," IEEE Transactions on Antennas and Propagation, vol. 48, no. 5, pp. 768-776, 2000.

[30] A. H. E. Zooghby, H. L. Southall, and C. G. Christodoulou, "Experimental validation of a neural network direction finder,"vol. 3, pp. 1592-1595, in Proceedings of the IEEE Antennas and Propagation Society International Symposium 1999 Digest, vol. 3, pp. 1592-1595, IEEE, Orlando, FL, USA, July 1999. 
[31] A. H. E. Zooghby, C. G. Christodoulou, and M. Georgiopoulos, "Multiple Sources neural network direction finding with arbitrary separations," in Proceedings of the 1998 IEEE-APS Conference on Antennas and Propagation for Wireless Communications, pp. 57-60, IEEE, Waltham, MA, USA, November 1998.

[32] A. H. E. Zooghby, C. G. Christodoulou, and M. Georgiopoulos, "Performance of radial-basis function networks for direction of arrival estimation with antenna arrays," IEEE Transactions on Antennas and Propagation, vol. 45, no. 11, pp. 1611-1617, 1997.

[33] A. H. E. Zooghby, C. G. Christodoulou, and M. Georgiopoulos, "Antenna array signal processing with neural networks for direction of arrival estimation," vol. 4, pp. 2274-2277, in Proceedings of the IEEE Antennas and Propagation Society International Symposium 1997 Digest, vol. 4, IEEE, Montreal, Canada, July 1997.

[34] H. M. Pour, Z. Atlasbaf, A. Mirzaee, and M. Hakkak, "A hybrid approach involving artificial neural network and ant colony optimization for direction of arrival estimation," in Proceedings of the 2008 Canadian Conference on Electrical and Computer Engineering, May 2008.

[35] P. Zhang, "DoA estimation method based on neural network," in Proceedings of the 2015 10th International Conference on P2P, Parallel Grid, Cloud and Internet Computing, pp. 828831, IEEE, Krakow, Poland, November 2015.

[36] M. F. Unlersen and E. Yaldiz, "Direction of arrival estimation by using artificial neural networks," in Proceedings of the 2016 European Modelling Symposium, IEEE, Pisa, Italy, pp. 242245, November 2016.

[37] J. S. Jeong, K. Araki, and J.-I. Takada, “A neural network for direction of arrival estimation under coherent multiple waves," in Proceedings of the 1998 IEEE Asia-Pacific Conference on Circuits and Systems, Micoelectronics and Integrating Systems, pp. 495-498, IEEE, Chiangmai, Thailand, November 1998.

[38] X. Tong, H. Wang, and Z. Gan, "Satellite interference location based on RBF neural network method,"vol. 1, pp. 445-449, in Proceedings of the 2000 5th International Conference on Signal Processing, vol. 1, pp. 445-449, IEEE, Beijing, China, August 2000.

[39] C. S. Lee, "Non-linear adaptive techniques for DoA estimation: a comparative analysis," in Proceedings of the Electronic Technology Directions to the Year 2000, pp. 72-77, IEEE, Adelaide, Australia, May 1995.

[40] H. Chen, B. Li, and Z. Shen, "Efficient network training method for two-dimensional DoA estimation," in Proceedings of the the Fourth International Conference on Computer and Information Technology, pp. 1028-1032, IEEE, Wuhan, China, September 2004.

[41] J. D. Ndaw, A. Faye, and A. S. Maiga, "Decoupled 2D DOA estimation using LVQ neural networks and UCA arrays," in Proceedings of the 2016 IEEE Radio and Antenna Days of the Indian Ocean (RADIO), pp. 1-2, IEEE, St. Gilles-les-Bains, Reunion, October 2016.

[42] T. Matsumoto and Y. Kuwahara, "Experiments of direction finder by RBF neural network with post processing," vol. 4A, pp. 10-13, in Proceedings of the 2005 IEEE Antennas and Propagation Society International Symposium, vol. 4A, pp. 10-13, IEEE, Washington, DC, USA, July 2005.

[43] N. C. Raj, P. V. Aswathy, and K. V. Sagar, "Determination of angle of arrival using nonlinear support vector machine regressors," in Proceedings of the IEEE-ICSCN, pp. 512-515, IEEE, Chennai, India, February 2007.

[44] K. A. Gotsis, K. Siakavara, and J. N. Sahalos, "On the direction of arrival (DoA) estimation for a switched-beam antenna system using neural networks," IEEE Transactions on Antennas and Propagation, vol. 57, no. 5, pp. 1399-1411, 2009.

[45] K. A. Gotsis, T. Kaifas, K. Siakavara, and J. Sahalos, "Direction of arrival estimation for a switched-beam DS-CDMA system using neural networks," in Proceedings of the 2007 19th International Conference on Applied Electromagnetics and Communications, pp. 1-4, IEEE, Dubrovnik, Croatia, September 2007.

[46] N. Fonseca, M. Coudyser, J.-J. Laurin, and J.-J. Brault, “On the design of a compact neural network-based DoA estimation system," IEEE Transactions on Antennas and Propagation, vol. 58 , no. 2, pp. 357-366, 2010.

[47] K. George and K. S. Sajjanshetty, "Neural network architectures for time-varying direction-of-arrival estimation," in Proceedings of the 5th International Conference on Industrial and Information Systems, pp. 69-74, IEEE, Mangalore, India, July-August 2010.

[48] M. Agatonovic and Z. Stankovic, "Hybrid ANN model for accurate 2D DoA estimation of a radiating source," in Proceedings of the TELSIKS, pp. 370-373, IEEE, Nis, Serbia, October 2013.

[49] M. Agatonovic, Z. Stankovic, and B. Milovanovic, "High resolution two-dimensional DoA estimation using artificial neural networks," in Proceedings of the 2012 6th European Conference on Antennas and Propagation, pp. 1-5, IEEE, Prague, Czech Republic, March 2012.

[50] B. Milovanovic, M. Agatonovic, Z. Stankovic, N. Doncov, and M. Sarevska, "Application of neural networks in spatial signal processing," in Proceedings of the 11th Symposium on Neural Network Applications in Electrical Engineering, pp. 5-14, IEEE, Belgrade, Serbia, September 2012.

[51] M. Agatonovic, Z. Stankovic, B. Milovanovic, and N. Doncov, "DoA estimation using radial basis function neural networks as uniform circular antenna array signal processor," in Proceedings of the TELSIKS, pp. 544-547, IEEE, Nis, Serbia, October 2011.

[52] S. Caylar, "A new neural network DoA estimation technique based on subarray beamforming," in Proceedings of the 2009 International Conference on Electromagnetics in Advanced Applications, pp. 732-734, IEEE, Torino, Italy, September 2009.

[53] S. Caylar, K. Leblebicioglu, and G. Dural, "A neural network method for direction of arrival estimation with uniform circular dipole array in the presence of mutual coupling," in Proceedings of the 20073 rd International Conference on Recent Advances in Space Technologies, pp. 537-540, IEEE, Istanbul, Turkey, June 2007.

[54] Faye, A. B. Youm, and J. D. Ndaw, "LVQ based DoA estimation," in Proceedings of the 2013 fifth International Conference on Computational Intelligence, Communication Systems and Networks, pp. 245-250, Madrid, Spain, June 2013.

[55] Y. L. Sit, M. Agatonovic, and T. Zwick, "Neural network based direction of arrival estimation for a MIMO OFDM Radar," in Proceedings of the 2012, Proceedings of the 9th European Radar Conference, IEEE, Amsterdam, Netherlands, pp. 298-301, October-November 2012.

[56] Z. Stankovic, N. Doncov, J. Russer, T. Asenov, and B. Milovanovic, "Efficient DoA estimation of impinging stochastic EM signal using neural networks," in Proceedings of the 2013 International Conference on Electromagnetics in Advanced Applications, pp. 575-578, IEEE, Torino, Italy, September 2013.

[57] Z. Stankovic, N. Doncov, B. Milovanovic, and J. Russer, "Neural network approach for efficient DoA determination of 
multiple stochastic EM Sources in far-field," in Proceedings of the 2014 International Conference on Numerical Electromagnetic Modeling and Optimization for RF, Microwave, and Terahertz Applications, pp. 1-4, IEEE, Pavia, Italy, May 2014.

[58] Z. Stankovic, N. Doncov, I. Milovanovic, and B. Milvanovic, "Neural network model for efficient localization of a number of mutually arbitrary positioned stochastic EM sources in farfiled," in Proceedings of the 2014 12th Symposium on Neural Network Applications in Electrical Engineering, November 2014.

[59] Z. Stankovic, N. Doncov, B. Milovanovic, J. Russer, I. Milovanovic, and M. Agatonovic, "Neural networks-based DoA estimation of multiple stochastic narrow-band EM sources,"vol. 2, pp. 526-529, in Proceedings of the TELSIKS, vol. 2, pp. 526-529, IEEE, Nis, Serbia, October 2013.

[60] S. Chakrabarty and E. A. P. Habets, "Broadband DoA estimation using convolutional neural networks trained with noise signals," in Proceedings of the 2017 IEEE workshop on applications of signal processing to audio and acoustics, pp. 136-140, IEEE, New Paltz, NY, USA, October 2017.

[61] Faye, J. D. Ndaw, and A. S. Maiga, "Two-dimensional DoA estimation based on a single uniform linear array," in Proceedings of the 25th Telecommunications forum, pp. 1-4, IEEE, Belgrade, Serbia, November 2017.

[62] E. Efimov, T. Shevgunov, and D. Filimonova, "Angle of arrival estimator based on artificial neural network," in Proceedings of the 2016 17th International Radar Symposium, pp. 1-3, IEEE, Krakow, Poland, May 2016.

[63] H. Huang, J. Yang, H. Huang, Y. Song, and G. Gui, “Deep learning for super-resolution channel estimation and DOA estimation based massive MIMO system," IEEE Transactions on Vehicular Technology, vol. 67, no. 9, pp. 8549-8560, 2018.

[64] J. J. Tong and Y. F. Fang, "Robust sound localization of sound sources using deep convolution network," in Proceedings of the 2019 ICCA, pp. 196-200, IEEE, Edinburgh, UK, July 2019.

[65] Y. M. Wang and Y. L. Ma, "The performance of neural network for high resolution direction-of-arrival estimation," in Proceedings of the China 1991 International Conference on Circuit and Systems, pp. 301-304, IEEE, Shenzhen, China, June 1991.

[66] Y. Chen and C. Hou, "High resolution adaptive bearing estimation using a complex-weighted neural network," in Proceedings of the IEEE International Conference on Acoustics, Speech, and Signal Processing, pp. 317-320, IEEE, San Francisco, CA, USA, March 1992.

[67] S.-H. Chang, T.-Y. Lee, and W.-H. Fang, "High-resolution bearing estimation via unitary decomposition artificial neural network (UNIDANN)," in Proceedings of the IEEE International Conference on Acoustics, Speech, and Signal Processing, pp. 3607-3610, IEEE, Detroit, MI, USA, May 1995.

[68] Y. Guo, Z. Zhang, Y. Huang, and P. Zhang, "DOA estimation method based on cascaded neural network for two closely spaced sources," IEEE Signal Processing Letters, vol. 27, pp. 570-574, 2020. 